\title{
Mechanical Thrombectomy Using the Solitaire FR system for Occlusion of the Top of the Basilar Artery: Intentional Detachment of the Device after Partial Retrieval
}

\author{
Kwong Lok Siu, MD², Dong-Geun Lee, MD', Jae Ho Shim, MD', \\ Dae Chul Suh, MD', Deok Hee Lee, MD'
}

Acute, distal, basilar artery occlusion is a challenging neurovascular emergency. There have been several reports regarding the successful application of the Solitaire FR device for treating this lesion. However, due to the lack of a suitable, balloon-tipped, guiding catheter for the vertebral artery, during this procedure we frequently experience the occurrence of clot fragmentation and distal migration. There may be some technical solutions to solve this problem. The purpose of this report is to present a technical variation of using the Solitaire FR, and which is referred to as the 'intentional device detachment technique.' As a clot tends to re-embolize during its passage through the tortuous cranio-cervical junction level of the vertebral artery or its passage through the tip of the guiding catheter, due to the lack of proximal flow arrest, we thought that not removing the stent segment of the device which is capturing the clot could avoid this problem. We were able to successfully apply this technique in two cases. We believe that this technique can be a possible technical option for using the Solitaire FR device when a patient has little concern regarding the subsequent use of antiplatelets.

Key Words : Stroke/therapy; Basilar artery; Mechanical thrombolysis; Stent retriever

\footnotetext{
'Department of Radiology, Research Institute of Radiology, Asan Medical Center, University of Ulsan College of Medicine, Seoul, Korea; '2Department of Radiology, Tuen Mun Hospital, Tsing Chung Koon Road, Tuen Mun, New Territories, Hong Kong

Received January 24, 2014; accepted after revision February 18, 2014.

Correspondence to: Deok Hee Lee, MD, PhD, Department of Radiology, Research Institute of Radiology, University of Ulsan College of Medicine, Asan Medical Center, 86 Asanbyeongwon-gil, Songpa-gu, Seoul 138-736, Korea.

Tel. 82.2.3010.5944 Fax. 82.10.6824.5944

E-mail:dhlee@amc.seoul.kr

This is an Open Access article distributed under the terms of the Creative Commons Attribution Non-Commercial License (http://creativecommons.org/licenses/by-nc/3.0) which permits unrestricted non-commercial use, distribution, and reproduction in any medium, provided the original work is properly cited.
}

Stent retrievers, such as the Solitaire FR system (SFR) (ev3, Inc., Irvine, CA, USA), have become the primary endovascular thrombectomy device together with the aspiration thrombectomy catheter. The SFR device has also been successfully used for the treatment of acute basilar artery occlusion (BAO), with the reported recanalization rates ranging from $74 \%$ to 94.4\% [1-3]. However, our experience differed slightly from those reports. In our earlier experience with SFR, it was quite difficult to completely retrieve a clot even after multiple passages of the device.

In a typical BAO situation, we could observe antegrade flow through the inner lumen of the device as soon as the device was placed across the occluded 
segment. We could also note the filling defect which was displaced by the mesh. After each passage of the device, we could observe slight displacement of the filling defects and occasionally we experienced distal migration of the fragmented clots.

After several attempts, we determined that the cause of refractoriness was due to re-embolization of the almost retrieved clots down to the $\mathrm{C} 1-2$ vertebral body level proximally or to the tip of the guiding catheter level. As the guiding catheter was not balloon-tipped, the retrieving clots were easily detached from the mesh and migrated back to the original occlusion site which was the top of the basilar artery. We also attempted vigorous aspiration of the guiding catheter during retrieval, although this was unsuccessful.

As there is no currently available, balloon-tipped guiding catheter specifically designed for the vertebral artery, it is quite frustrating to repeat unsuccessful passages without achieving flow arrest. In this situation, switching to an aspiration thrombectomy catheter can be considered, however, this method is also not free from the same problem without the aid of the proximal flow control system.

The purpose of this report is to present a technical variation using SFR, i.e. the 'intentional stent detachment technique', which could be one of the options to overcome the distal migration problem in the endovascular treatment of acute BAO. As our first patient indicated the feasibility of the technical variation, we thus applied the technique from the beginning of the procedure in the second patient.

\section{Patient 1}

A 67-year-old male presented in our emergency department complaining sudden, severe dizziness and left ptosis of an hour's duration. On admission, his symptoms gradually worsened, showing left-side facial palsy, severe dysarthria, and left-limb ataxia. He was on oral anticoagulation with a daily aspirin since he had a mechanical aortic valve replaced more than a year previously. However, his international normalization ratio (INR) on admission turned out to be only 1.4.

Subsequent, acute stroke MR imaging showed occlusion of the basilar artery with a focal infarction in the left superior cerebellar artery territory (Fig. 1A). Systemic tissue plasminogen activator (Actylase, Boehringer Ingelheim, Germany) was given at a dose of $0.6 \mathrm{mg} / \mathrm{kg}$ as the patient was still within the time window of intravenous thrombolytic therapy. Because the patient did not show any symptomatic improvement, our endovascular treatment team was called in per our institutional acute stroke bridging therapy protocol.

Right vertebral angiogram showed a typical pattern of BAO. The lesion showed antegrade flow directly after placement of the SFR (Fig. 1B). However, the first retrieval was unsuccessful and only showed a slight change in the shape of the filling defect. The pattern repeated during several, following trials. Eventually, the clot migrated further distally into the left posterior cerebral artery (Fig. 1C).

At the time of the final device retrieval, we obtained a control angiogram while the device was still in the midportion of the basilar trunk (Fig. 1D). We were able to retract the filling defect proximally into the mid portion of the basilar trunk. However, it was attached at the distal tip of the device. Eventually we could pull down the device to the distal vertebral artery (VA) level proximal to the ipsilateral posterior inferior cerebellar artery (PICA) origin.

On the control angiogram we noted that half of the filling defect had migrated into the right PCA and half of it was still within the device mesh as we thought that further manipulation of the device would result in additional distal migration of the clot, we decided to purposely detach the device at the current position in order to avoid the risk of another distal migration (Fig. $1 \mathrm{E})$.

A loading dose of tirofiban (Aggrastat, Iroko Cardio Australia Pty Ltd, Australia) was given systemically on the table. On the following day, the patient's symptoms improved significantly and only showed mild residual diplopia on the left lateral gaze. A dual antiplatelet regimen was initiated on top of oral anticoagulation.

Follow-up MR showed no new infarcts even in the right PCA territory. There was also no evidence of any hemorrhagic transformation. The patient was discharged with instructions to take both aspirin and warfarin after adjustment of the INR.

\section{Patient 2}

A 64-year-old male presented with altered mental status. He was found unconscious and his last known normal time period was four hours previously. His initial NIHSS stroke score was 18 . He did not have any known stroke risk factors. However, atrial fibrillation was seen at the time of his admission. Acute stroke MR revealed multi-territorial, wedge-shaped infarcts involving both cerebellar hemispheres and the left PCA territory. Subtle signal changes were also noted in the pons.

An urgent, right vertebral angiogram showed distal 


\section{Kwong Lok Siu, et al.}
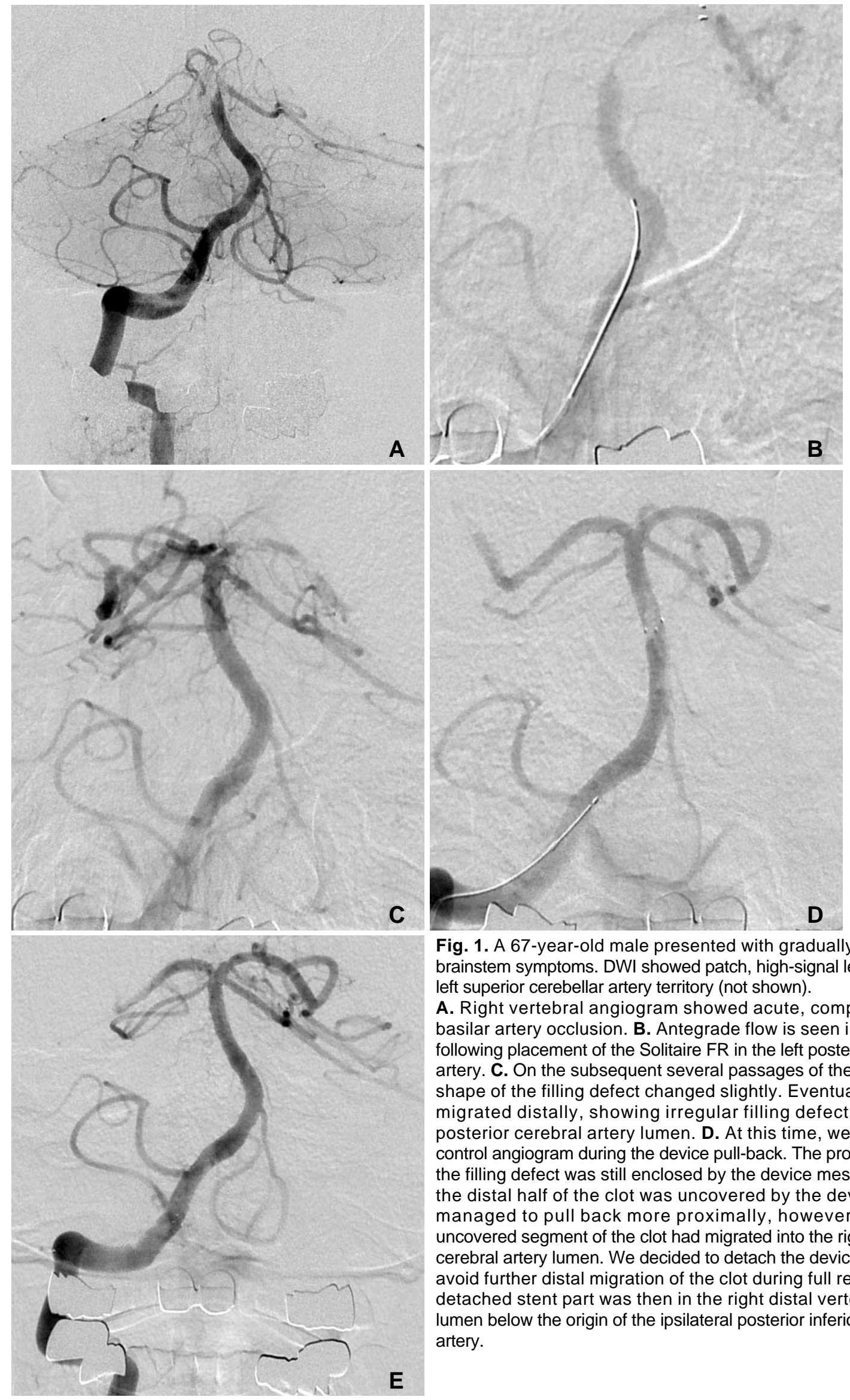

Fig. 1. A 67-year-old male presented with gradually worsening brainstem symptoms. DWI showed patch, high-signal lesions in the left superior cerebellar artery territory (not shown).

A. Right vertebral angiogram showed acute, complete, distal basilar artery occlusion. B. Antegrade flow is seen immediately following placement of the Solitaire FR in the left posterior cerebral artery. C. On the subsequent several passages of the device, the shape of the filling defect changed slightly. Eventually, the clot migrated distally, showing irregular filling defects in the left posterior cerebral artery lumen. D. At this time, we obtained a control angiogram during the device pull-back. The proximal half of the filling defect was still enclosed by the device mesh, however, the distal half of the clot was uncovered by the device. E. We managed to pull back more proximally, however, the distal uncovered segment of the clot had migrated into the right posterior cerebral artery lumen. We decided to detach the device in order to avoid further distal migration of the clot during full retrieval. The detached stent part was then in the right distal vertebral artery lumen below the origin of the ipsilateral posterior inferior cerebellar artery. 

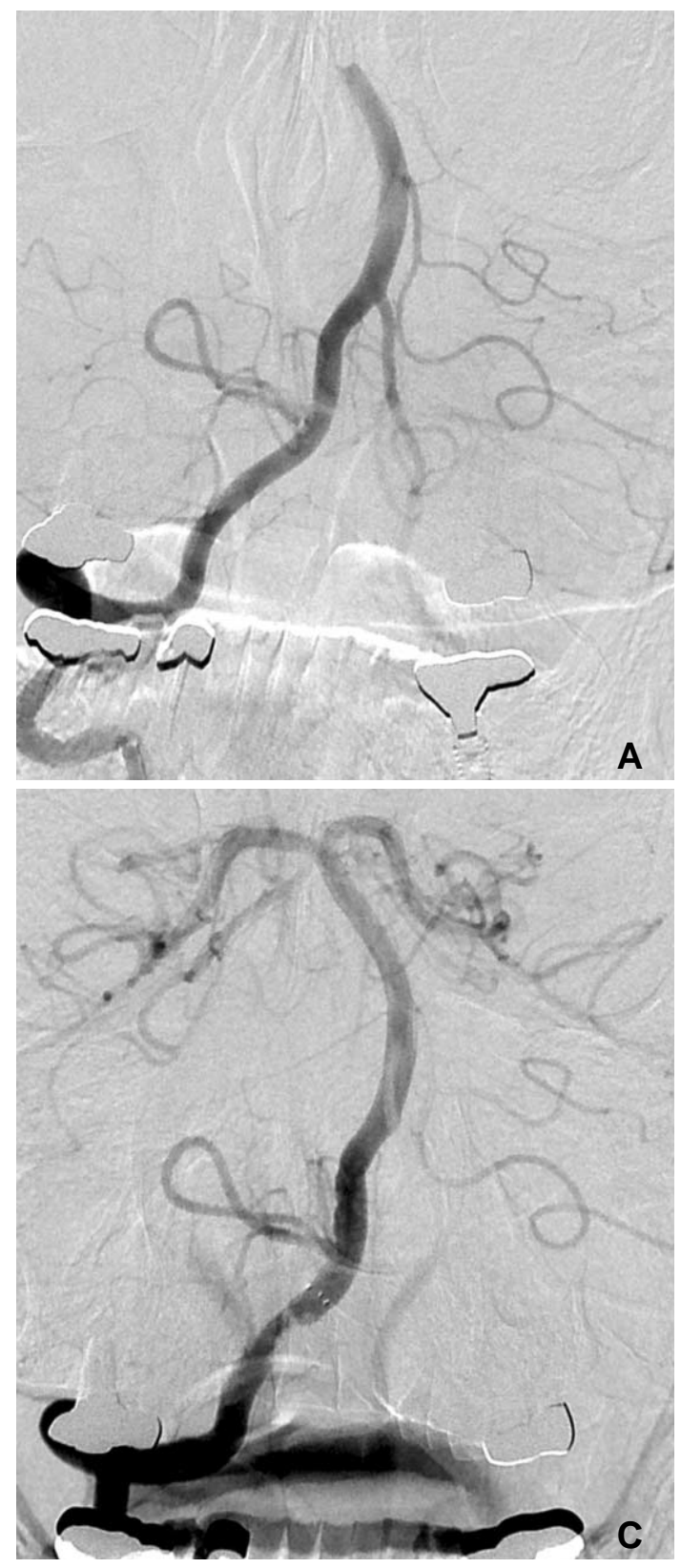

BAO with a filling defect showing a meniscus sign. (Fig. 2A) An oval-shaped filling defect was noted along the open arterial lumen when the SFR was placed (Fig. 2B). We could pull down the device with the filling defect down to the distal VA level below the origin of the PICA without any fragmentation and/or migration of the clot distally. We decided to detach the stent part at that level (Fig. 2C).

As the volume of the initial infarct was significant, aspirin monotherapy was started without anti-coagulation which could not be initiated due to combined gastro-intestinal tract bleeding during the acute phase. On his discharge from the intensive care unit, the

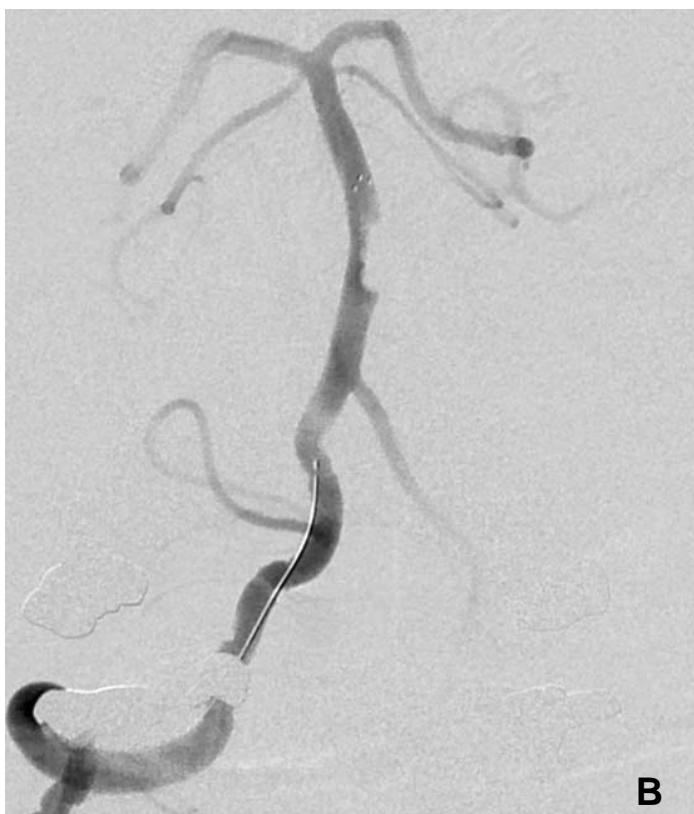

Fig. 2. A 64-year-old male presented with altered mental status. The initial DWI showed multifocal cerebellar hemispheric and brainstem infarcts (not shown).

A. Right vertebral angiogram showed complete occlusion of the basilar top. B. The artery was open as soon as the device was placed. The control angiogram taken while the device was pulled back, shows a well-marginated filling defect. C. The device was intentionally detached. The clot was still caught by the device while the artery was fully open and without distal clot migration.

patient was placed on aspirin and warfarin. After rehabilitation, he could walk with some assistance. His modified Rankin score at 90 days was 3.

\section{DISCUSSION}

The SFR is a self-expanding stent retriever designed for mechanical thrombectomy and restoration of blood flow in patients with acute ischemic stroke. The recommended usage is in conjunction with at least an 8-French balloon guide sheath and aspiration, so as to reduce the risk of clot fragmentation and distal embolization during device retrieval. However, for 


\section{Kwong Lok Siu, et al.}

vertebrobasilar application, the small size and tortuosity of the vertebral artery makes cannulation very difficult. Due to this inherent limitation, clot fragmentation and migration occur quite frequently during use of stent retrievers in patients with BAO.

Then how to cope with this problem? There could be several possible solutions. Attempt flow arrest using a balloon-tipped catheter of a smaller caliber (O.D.), which is, however, not currently possible due to its unavailability. We were informed that there is a 6-Fr., balloon-tipped guiding catheter made by Fuji Systems Corp. (Cello; Tokyo, Japan). This could be the best solution, however, due to the limitation of the inner diameter, there might be some limitation in its ability to aspirate.

Another possible solution is the use of another type of thrombectomy device, such as the Penumbra aspiration device (Penumbra, Inc., Alameda, CA, USA) or the Merci device (Concentric Medical, Mountain View, CA, USA). However, we do not believe that anyone would go back to the Merci device again As, actually, the reported successful recanalization rate using the Merci device is only $57.3 \%$ and with a mortality rate of $34 \%[4,5]$. Therefore, the penumbra aspiration catheter should be selected as the first-line device for distal $\mathrm{BAO}$ as the reported successful recanalization rate is $81.6 \%$ with the 90 -day mortality rate being $32.8 \%$ [ 6 , 7]. However, as we previously mentioned, that device also has the problem of clot fragmentation and migration unless it has the assistance of proximal flow arrest. Use of the catheter together with SFR could also be another option. Reported cases have suggested as one of the useful options for mechanical thrombectomy in acute $\mathrm{BAO}[8]$.

The bilateral vertebral artery approach could be another solution. We can retrieve the device catching the clot below the vertebrobasilar junction (VBJ) and insert a compliant occlusion balloon into the basilar trunk via the contralateral VA. By occluding the VBJ, we can protect the artery from distal migration while removing the device and clot through the guiding catheter. This technique cannot be performed in a unilateral or hypoplastic VA.

In addition to those options, we would like to add to the list this technical variation of using SFR. We believe this 'intentional device detachment technique' has several advantages over other methods. For example, we can minimize the procedure time and can achieve antegrade flow as soon as the device is placed and then left open afterwards. As the device was pulled back just once, we can thus minimize potential endothelial damage. Most of all, this technique may minimize the risk of clot fragmentation and subsequent distal embolization which occurs quite frequently when using the current techniques.

However, as we experienced in our patients, the requirement for antiplatelet therapy due to the residual stent segment, could be the most significant disadvantage of this technique. When the infarct volume is not large, as it was in our patient 1 , the use of dual antiplatelets would not pose a significant problem. However, if the volume is large, as it was in our patient 2 , it would pose a difficult clinical decision-making issue due to the potential risk of hemorrhagic transformation. Moreover, as both of our patients were, the clinical decision would be much more difficult when a patient required anticoagulation, and which could be quite common in acute stroke patients. However, since many clinicians have already experienced successful use of various, self-expanding stents for the treatment of acute ischemic stroke before the stent retriever became available, this technique could be an option for this particular situation of $\mathrm{BAO}$ [9].

Clot fragmentation and distal migration frequently occurs during application of the Solitaire FR system for acute BAO due to the lack of proximal flow arrest during retrieval. The problem can be eliminated using the 'intentional device detachment technique' which can minimize the time to recanalization and thus easily reduce the potential for endothelial damage.

\section{References}

1. Mourand I, Machi P, Milhaud D, Picot MC, Lobotesis K, Arquizan C, et al. Mechanical thrombectomy with the Solitaire device in acute basilar artery occlusion. J Neurointerv Surg 2013 May 4. [Epub ahead of print]

2. Espinosa de Rueda M, Parrilla G, Zamarro J, García-Villalba B, Hernández F, Moreno A. Treatment of acute vertebrobasilar occlusion using thrombectomy with stent retrievers: initial experience with 18 patients. AJNR Am J Neuroradiol 2013;34:10441048

3. Mordasini P, Brekenfeld C, Byrne JV, Fischer U, Arnold M, Heldner MR, et al. Technical feasibility and application of mechanical thrombectomy with the Solitaire FR Revascularization Device in acute basilar artery occlusion. AJNR Am J Neuroradiol 2013;34:159-163

4. Noorian AR, Gupta R, Nogueira RG. Acute stroke: techniques and results with the Merci retriever. Tech Vasc Interv Radiol 2012;15:47-52

5. Smith WS, Sung G, Saver J, Budzik R, Duckwiler G, Liebeskind DS, et al. Mechanical thrombectomy for acute ischemic stroke: final results of the Multi MERCI trial. Stroke 2008;39:1205-1212

6. Liu W, Kung DK, Mahaney KB, Rossen JD, Jabbour PM, Hasan DM. Anterior-to-posterior circulation approach for mechanical thrombectomy of an acutely occluded basilar artery using the 


\section{Thrombectomy for Occlusion of the Top of the Basilar Artery}

penumbra aspiration system. World Neurosurg 2012;77:398

7. Penumbra Pivotal Stroke Trial Investigators. The penumbra pivotal stroke trial: safety and effectiveness of a new generation of mechanical devices for clot removal in intracranial large vessel occlusive disease. Stroke 2009;40:2761-2768

8. Deshaies EM. Tri-axial system using the Solitaire-FR and
Penumbra Aspiration Microcatheter for acute mechanical thrombectomy. J Clin Neurosci 2013;20:1303-1305

9. Kim SM, Lee DH, Kwon SU, Choi CG, Kim SJ, Suh DC. Treatment of acute ischemic stroke: feasibility of primary or secondary use of a self-expanding stent (Neuroform) during local intra-arterial thrombolysis. Neuroradiology 2012;54:35-41 